\title{
Heavy and light hadron spectroscopy on the lattice
}

\author{
Christopher E. Thomas* (for the Hadron Spectrum Collaboration) \\ DAMTP, Univ. of Cambridge \\ E-mail: c.e.thomas@damtp.cam.ac.uk
}

\begin{abstract}
I report on some recent progress in studying heavy and light hadrons using lattice QCD. Results from calculations of excited charmonia and open-charm mesons are discussed where novel techniques have enabled the extraction of extensive spectra, including states with exotic quantum numbers and non-exotic hybrids, with high statistical precision; the interesting phenomenology suggested by these results will be highlighted. I then discuss investigations of unstable states on the lattice, concentrating on a study which mapped out the energy-dependence of the $\rho$ resonance in $\pi \pi$ scattering. Computations of spectra of excited light, strange and charmed baryons are also mentioned and some implications of the results discussed.
\end{abstract}

XV International Conference on Hadron Spectroscopy

4-8/11/2013

Nara, Japan

${ }^{*}$ Speaker. 


\section{Introduction}

Over the last decade there has been renewed interest in hadron spectroscopy driven by numerous puzzling experimental observations. As we have heard at this meeting, many of these are the subject of active discussion and current and planned experimental investigations. For example, there are various "X,Y,Z's" in charmonium and charged charmonium and bottomonium-like structures. Then there are other questions such as the nature of the first excitation of the nucleon (the Roper) and the light scalar mesons, and 'missing' baryon resonances - have these just not been observed (perhaps because they are too wide or they couple weakly to the production mechanisms in current experiments) or are some degrees of freedom 'frozen' in baryons.

There has been much speculation as to the nature of these unexpected structures and various QCD-inspired approaches have been used in attempts to describe their properties. For example, are some tetraquarks, molecular states of hadrons, hadro-charmonia or hybrid mesons where the gluonic field is excited? States with exotic quantum numbers, i.e. those that cannot arise from solely a quark-antiquark pair, are particularly interesting because they are a smoking gun for physics beyond a simple quark model. For example, exotic spin $(J)$, parity $(P)$, charge-conjugation (C) combinations (e.g. $J^{P C}=0^{--}, 0^{+-}, 1^{-+}, 2^{+-}$) or exotic flavour states (e.g. charmonium and bottomonium-like states with non-zero charge).

To properly address these questions, test QCD and understand its low-energy regime, we must make ab-initio calculations in QCD. Lattice QCD provides a method for performing such computations in the non-perturbative regime: four-dimensional space-time is discretised on a finite four-dimensional hypercubic lattice with a Euclidean (imaginary-time) metric. Quantities of interest are then computed in the path integral formulation using importance-sampling Monte Carlo methods. Calculations of the low-lying spectrum of hadrons have long been benchmarks of lattice methods but only in the last few years has there been significant progress in using lattice QCD to study excited hadrons and, even more recently, in investigating unstable and near-threshold states. In these proceedings I briefly summarise the results from some of the Hadron Spectrum Collaboration's recent work. A more general review of recent lattice results for hadron spectroscopy can be found in Ref. [1]. I begin in Section 2 with computations of spectra of excited charmonia and opencharm mesons and then discuss a study of the energy dependence of the $\rho$ resonance in Section 3. I comment on calculations of excited baryon spectra in Section 4 before concluding in Section 5 .

\section{Excited charmonia and open-charm mesons}

Over the last few years, through the development of a combination of novel techniques, we have made significant progress in using lattice QCD to compute spectra of excited light isovector [5, 6] and isoscalar [7, 8] mesons, charmonia[2] and open-charm mesons[4]. Figs. 1 and 2 show, respectively, the spectra of excited charmonia and charm-light mesons from lattice QCD calculations with dynamical strange and degenerate up and down quarks corresponding to $M_{\pi} \approx 400 \mathrm{MeV}$. The lattice is anisotropic with a temporal lattice spacing, $a_{t}$, finer than the spatial lattice spacing, $a_{s} \approx 0.12 \mathrm{fm}$, and $\xi=a_{s} / a_{t} \approx 3.5$, and the spatial volume is $24^{3}$ in lattice units corresponding to a spatial extent $L_{s} \approx 2.9 \mathrm{fm}$. Only connected contributions to the charmonium correlators are 


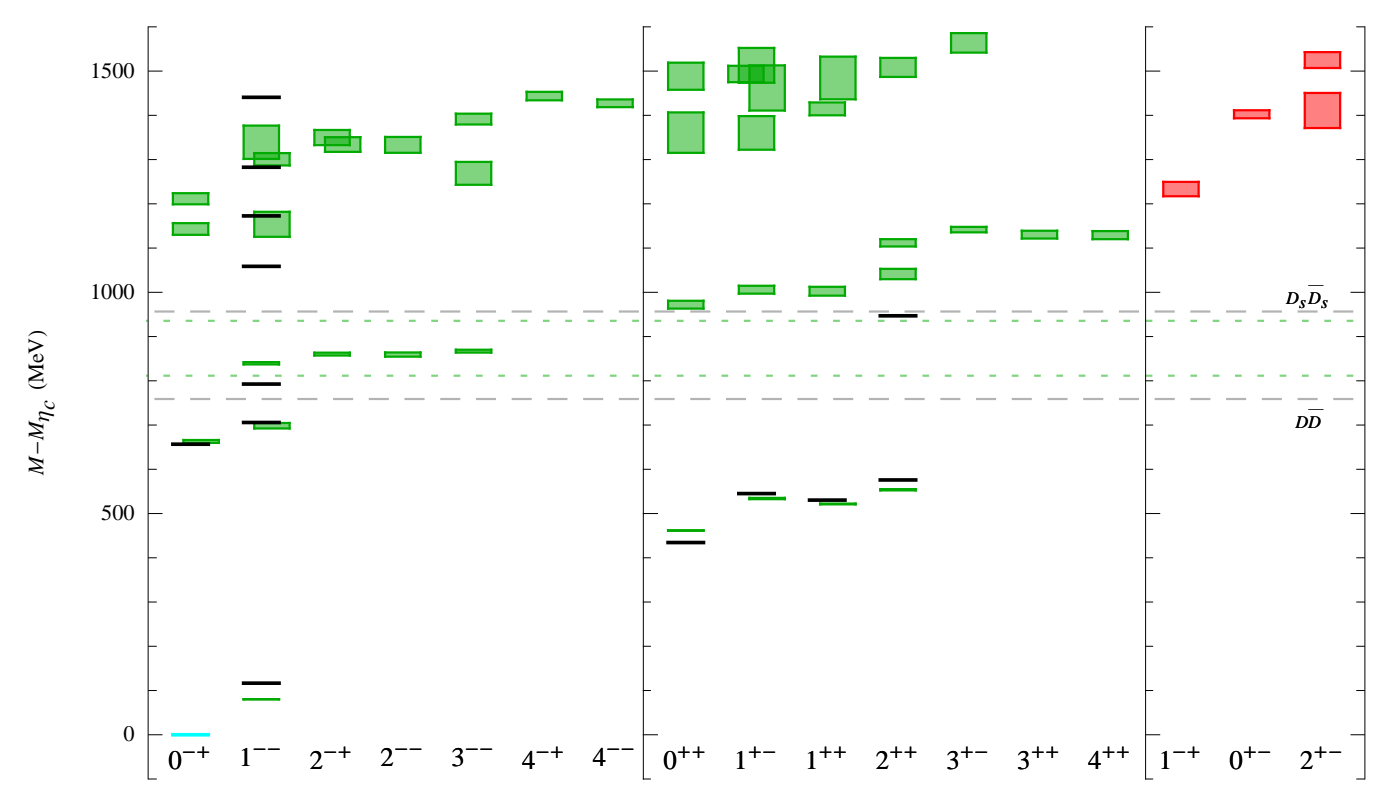

Figure 1: From Ref. [2]. Summary of the charmonium spectrum labelled by $J^{P C}$. The red and green boxes are the calculated masses (with $M_{\pi} \approx 400 \mathrm{MeV}$ ) and black lines are experimental values from the 2011 PDG summary tables [3]. The calculated (experimental) masses are shown with the calculated (experimental) $\eta_{c}$ mass subtracted. The vertical size of the boxes represents the one sigma statistical uncertainty on either side of the mean. The dashed lines indicate the lowest non-interacting $D \bar{D}$ and $D_{s} \bar{D}_{s}$ levels using calculated $D$ and $D_{s}$ masses (fine green dashing) and using the experimental masses (coarse grey dashing).

included; full details of the calculations, a similar charm-strange $\left(D_{s}\right)$ meson spectrum and results from another lattice volume are given in Refs. [2, 4].

The computed charmonium spectrum includes many states with exotic $J^{P C}$ quantum numbers and these are shown in the right panel of Fig. 1; by considering the overlaps of states with interpolating operators $[6,9]$ we identify these as hybrid mesons. In the non-exotic channels the majority of the states can be understood in terms of quark-model ${ }^{2 S+1} L_{J}$ multiplets ( $L$ is the orbital angular momentum and $S$ the total quark spin) but there are also some states that do not fit into this pattern; again, by considering operator-state overlaps, we identify these as non-exotic hybrids. We also identify hybrids in the open-charm meson spectra but because these mesons are not eigenstates of charge conjugation (or a generalisation) there are no exotic quantum numbers. In Refs. [2, 4] we highlight the states identified as hybrids and show that the pattern can be interpreted as a colouroctet quark-antiquark pair coupled to a $1^{+-}$chromomagnetic gluonic excitation. In each flavour sector the lightest gluonic excitation appears at an energy scale $\sim 1.2-1.3 \mathrm{GeV}$ above the lightest conventional meson. This pattern and energy scale are consistent with what was found in the light meson sector.

As discussed in Ref. [2], we do not see any clear evidence for multi-hadron states in our extracted spectra. The calculations used operators containing fermion bilinears; to reliably study multi-hadron states we need to enlarge the basis of operators to include those with more fermion fields, something I will return to in the next section. We note that states above threshold can have 


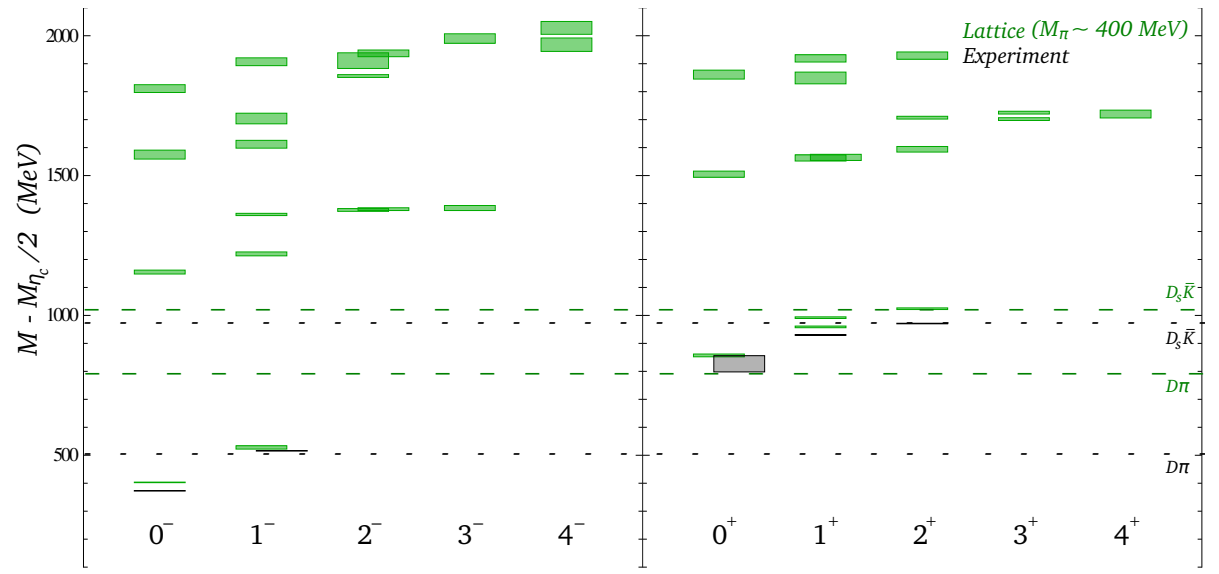

Figure 2: From Ref. [4]. As Fig. 1 but for the spectrum of charm-light $(D)$ mesons and shown with half the $\eta_{c}$ mass subtracted. The dashed lines indicate the lowest non-interacting $D \pi$ and $D_{s} \bar{K}$ levels using calculated meson masses (coarse green dashing) and using the experimental masses (fine black dashing).

large hadronic widths and a conservative approach is to only consider our current mass values accurate up to the hadronic width $[6,10]$.

\section{Energy-dependence of the $\rho$ resonance}

The vast majority of mesons can decay via the strong interaction to two or more lighter hadrons and many of the unexplained structures are near or above strong-decay thresholds. It is therefore essential to understand resonances and near-threshold states within QCD. However, in the Euclidean formulation of lattice QCD direct access to dynamical properties such as the width of resonances is lost. The Lüscher method [11] and its extensions allow, in principle, at least in certain cases, indirect access to the infinite volume scattering parameters from the multi-hadron spectrum in a finite volume. The Hadron Spectrum Collaboration has developed techniques to determine excited multi-hadron spectra with a high statistical precision using carefully constructed multi-hadron interpolating operators [12]. Extensions of the Lüscher method can then be used to determine scattering phase shifts. In a first investigation we determined the $\pi \pi$ isospin-2 $S(L=0)$ and $D$-wave $(L=2)$ scattering phase shifts at a large number of kinematic points in the elastic region [12].

Recently we have applied these techniques to study one of the simplest resonances, the $\rho(770)$ appearing in $\pi \pi$ scattering in $L=1$ with $J^{P C}=1^{--}$and isospin $I=1$ [10]. Fig. 3 summarises the results of this study; the energy-dependence of the phase shift has been mapped out in detail with far more points than obtained in previous lattice investigations (see [10] for references). The plot convincingly shows the rapid rise in the phase shift from $0^{\circ}$ through $90^{\circ}$ to $180^{\circ}$ that is expected in the presence of a single isolated resonance. Although the determined resonance mass is not too different from experiment, the width is significantly smaller - this is simply because the pion mass in this study is $\approx 400 \mathrm{MeV}$ and so the phase space for decay is much reduced; the coupling $g$ defined in the figure, where the phase space factor is removed, is more consistent with the experimental value. It could be argued that perhaps such a detailed determination of the phase shift curve is unnecessary for the relatively simple and well understood $\rho$ resonance. However, the ability to 


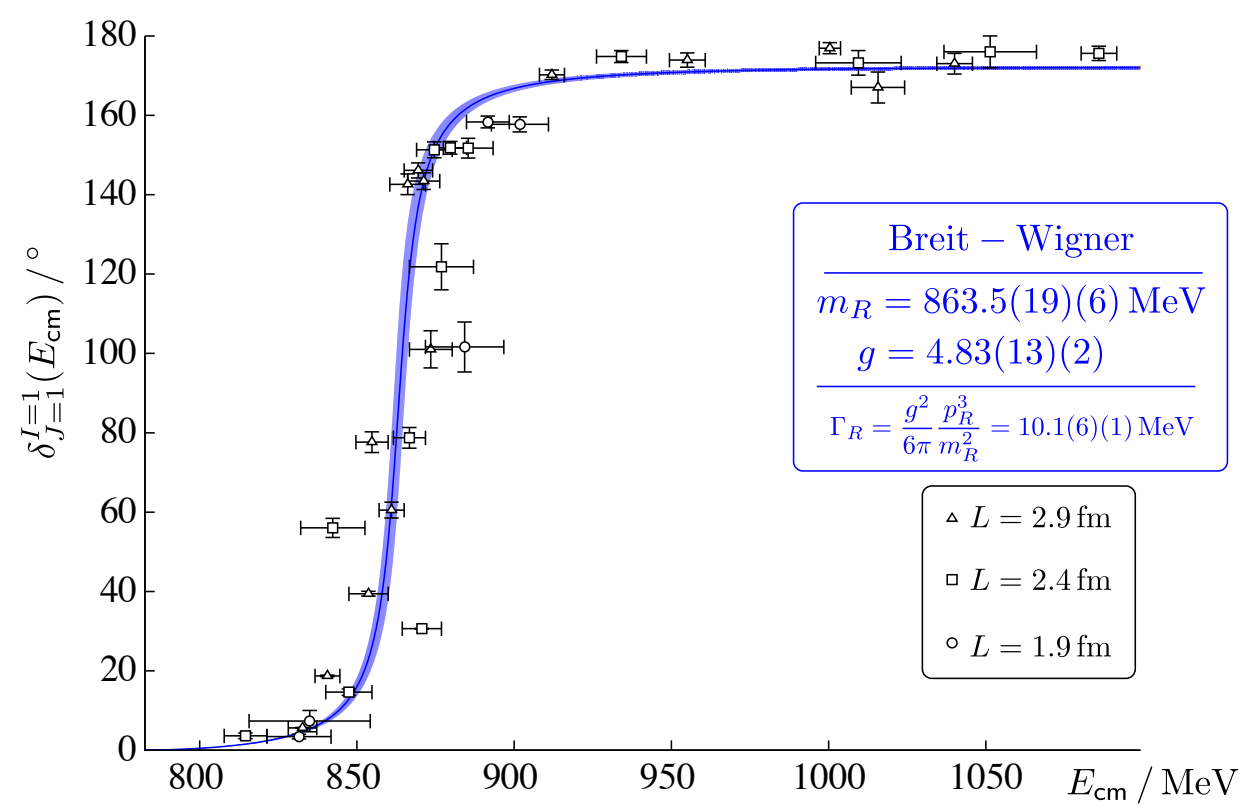

Figure 3: From [10]. The $I=1, L=1 \pi \pi$ elastic scattering phase shift plotted from $\pi \pi$ threshold to the inelastic $K \bar{K}$ threshold, from a dynamical calculation $\left(N_{f}=2+1\right)$ with $M_{\pi} \approx 400 \mathrm{MeV}$. Points are from an energy-level by energy-level analysis and the curve is from a global fit to a relativistic Breit Wigner parameterization as described in [10].

map out the energy dependence in detail will be vital in more complicated systems where the situation is phenomenologically less clear. I refer to a recent conference review, Ref. [1], for a discussion of some lattice studies of resonances and near-threshold states in other channels.

\section{Excited baryons}

Moving to baryons, the Hadron Spectrum Collaboration has recently computed very extensive excited spectra of light and strange baryons with all possible flavour combinations [13] and of charmed baryons $[14,15,16]$, extending earlier computations of non-strange $N$ and $\Delta$ baryon spectra $[17,18]$. Interpolating operators featuring three fermion fields were used and calculations were performed with the same lattice set up as above but with a different lattice volume (spatial extent $L_{s} \approx 1.9 \mathrm{fm}$ ). The computations of light and strange baryon spectra were performed for a range of light quark masses corresponding to $M_{\pi} \approx 700 \mathrm{MeV}$ (the SU(3) flavour symmetric point), 524 $\mathrm{MeV}$ and $400 \mathrm{MeV}$. As described in Ref. [13], this enabled the change in the spectra to be studied as the light (up and down) quark masses are reduced, i.e. SU(3) flavour symmetry is broken, and operator-state overlaps were used to investigate the mixing between $\mathrm{SU}(3)$ flavour basis states.

For each flavour sector and pion mass, the multiplicities of states in the lowest-energy bands are as would be expected from a non-relativistic quark model, i.e. the number of states from (flavour $\times$ spin $\times$ space) symmetry considerations - there does not appear to be any freezing of degrees of freedom. There are additional states within the spectra that have a large overlap with 
operators featuring the field strength tensor and, as discussed above, these are identified as hybrid hadrons. The $J^{P}$ pattern of the lightest hybrid baryons appears to be consistent with a colour-octet $1^{+-}$gluonic excitation coupled to colour octet $q q q$ and the scale of the excitation is $\sim 1.2-1.3$ $\mathrm{GeV}$. Interestingly this is the same pattern and energy scale as found in the studies of mesons with various flavours described above, suggesting common physics.

Another lattice investigation of excited light and strange baryons was presented at this meeting [19], a study of bottom ( $b b b$ ) baryons is described in Ref. [20] and for a more general review of baryon spectroscopy on the lattice I refer to Ref. [1].

\section{Outlook}

There has been significant progress in computing excited spectra on the lattice in recent years and calculations have given insight into the non-perturbative regime of $\mathrm{QCD}$, for example, the flavour structure of hadrons, the relevant low energy degrees of freedom and a phenomenology of hybrid hadrons. Building on these advances, much of the focus now is on understanding unstable and near-threshold hadrons. The relatively simple $\rho$ resonance has been studied in detail and, although first attempts have been made, there is still a lot of work to do for other channels. Through these investigations and studying other properties of hadrons on the lattice, for example photocouplings, the possibility that lattice QCD will be able to make further progress in addressing some of the open questions in spectroscopy in the near future looks very promising.

\section{Acknowledgments}

I thank the organisers of the conference for the opportunity to give this talk and my colleagues in the Hadron Spectrum Collaboration. Chroma [21] and QUDA [22, 23] were used to perform this work on the Lonsdale cluster maintained by the Trinity Centre for High Performance Computing funded through grants from Science Foundation Ireland (SFI), at the SFI/HEA Irish Centre for High-End Computing (ICHEC), and at Jefferson Laboratory under the USQCD Initiative and the LQCD ARRA project. Gauge configurations were generated using resources awarded from the U.S. Department of Energy INCITE program at the Oak Ridge Leadership Computing Facility at Oak Ridge National Laboratory, the NSF Teragrid at the Texas Advanced Computer Center and the Pittsburgh Supercomputer Center, as well as at Jefferson Lab. I acknowledge support for some of this work from a Marie Curie International Incoming Fellowship, PIIF-GA-2010-273320, within the 7th European Community Framework Programme.

\section{References}

[1] C. E. Thomas, Hadron spectroscopy review, PoS Lattice 2013 (2013) 003.

[2] L. Liu et. al. (for the Hadron Spectrum Collaboration), Excited and exotic charmonium spectroscopy from lattice QCD, JHEP 1207 (2012) 126, [arXiv:1204.5425].

[3] K. Nakamura et. al. (Particle Data Group), Review of particle physics, J.Phys.G G37 (2010) 075021. and 2011 partial update for the 2012 edition. 
[4] G. Moir, M. Peardon, S. M. Ryan, C. E. Thomas, and L. Liu, Excited spectroscopy of charmed mesons from lattice QCD, JHEP 1305 (2013) 021, [arXiv: 1301 . 7670 ].

[5] J. J. Dudek, R. G. Edwards, M. J. Peardon, D. G. Richards, and C. E. Thomas, Highly excited and exotic meson spectrum from dynamical lattice QCD, Phys. Rev. Lett. 103 (2009) 262001, [arXiv:0909.0200].

[6] J. J. Dudek, R. G. Edwards, M. J. Peardon, D. G. Richards, and C. E. Thomas, Toward the excited meson spectrum of dynamical QCD, Phys. Rev. D82 (2010) 034508, [arXiv: 1004 . 4930 ].

[7] J. J. Dudek et. al., Isoscalar meson spectroscopy from lattice QCD, Phys. Rev. D83 (2011) 111502, [arXiv:1102.4299].

[8] J. J. Dudek, R. G. Edwards, P. Guo, and C. E. Thomas, Toward the excited isoscalar meson spectrum from lattice QCD, Phys. Rev. D88 (2013) 094505, [arXiv:1309.2608].

[9] J. J. Dudek, The lightest hybrid meson supermultiplet in QCD, Phys. Rev. D84 (2011) 074023 , [arXiv:1106.5515].

[10] J. J. Dudek, R. G. Edwards, and C. E. Thomas, Energy dependence of the $\rho$ resonance in $\pi \pi$ elastic scattering from lattice QCD, Phys.Rev. D87 (2013) 034505, [arXiv:1212.0830].

[11] M. Luscher, Signatures of unstable particles in finite volume, Nucl. Phys. B364 (1991) 237-254.

[12] J. J. Dudek, R. G. Edwards, and C. E. Thomas, S and D-wave phase shifts in isospin-2 pi pi scattering from lattice QCD, Phys. Rev. D86 (2012) 034031, [arXiv:1203.6041].

[13] R. G. Edwards, N. Mathur, D. G. Richards, and S. J. Wallace, The Flavor Structure of the Excited Baryon Spectra from Lattice QCD, Phys.Rev. D87 (2013) 054506, [arXiv:1212 . 5236].

[14] M. Padmanath, R. G. Edwards, N. Mathur, and M. Peardon, Spectroscopy of triply-charmed baryons from lattice QCD, arXiv:1307.7022.

[15] M. Padmanath, R. G. Edwards, N. Mathur, and M. Peardon, Spectroscopy of doubly and triply-charmed baryons from lattice QCD, PoS Lattice 2013 (2013) 247, [arXiv: 1311.4354 ].

[16] M. Padmanath, R. G. Edwards, N. Mathur, and M. Peardon, Excited-state spectroscopy of singly, doubly and triply-charmed baryons from lattice QCD, arXiv:1311.4806.

[17] R. G. Edwards, J. J. Dudek, D. G. Richards, and S. J. Wallace, Excited state baryon spectroscopy from lattice QCD, Phys. Rev. D84 (2011) 074508, [arXiv:1104.5152].

[18] J. J. Dudek and R. G. Edwards, Hybrid Baryons in QCD, Phys.Rev. D85 (2012) 054016 , [arXiv:1201.2349].

[19] G. P. Engel, C. Lang, D. Mohler, and A. Schafer, Excited light and strange hadrons from the lattice with two Chirally Improved quarks, PoS Hadron 2013 (2013) 118, [arXiv: 1311. 6579].

[20] S. Meinel, Excited-state spectroscopy of triply-bottom baryons from lattice QCD, Phys.Rev. $\mathbf{D 8 5}$ (2012) 114510, [arXiv:1202.1312].

[21] R. G. Edwards and B. Joo (SciDAC Collaboration), The chroma software system for lattice qcd, Nucl. Phys. B. Proc. Suppl. 140 (2005) 832, [hep-lat/ 0409003 ].

[22] M. A. Clark, R. Babich, K. Barros, R. C. Brower, and C. Rebbi, Solving Lattice QCD systems of equations using mixed precision solvers on GPUs, Comput. Phys. Commun. 181 (2010) 1517-1528, [arXiv:0911.3191].

[23] R. Babich, M. A. Clark, and B. Joo, Parallelizing the QUDA Library for Multi-GPU Calculations in Lattice Quantum Chromodynamics, in International Conference for High Performance Computing, Networking, Storage and Analysis (SC), pp. 1-11, 2010. arXiv:1011.0024. 